\title{
Study of Centrifugal Filament Winding
}

\author{
Part 2: Mechanism of Degassing \\ By Yoshinori Nishino and Josuke Kawachi, Members, TMSJ \\ FRP Division, Hitachi Zosen Corporation, \\ Technical Research Institute, Hitachi Zosen Corporation, Konohana-ku, Osaka \\ Based on the Journal of the Textile Machinery Society of Japan, Vol. 37, No. 4, T58-T68 (1984-4)
}

\begin{abstract}
The centrifugal pipe forming method has an advantage of better resin impregnation and degassing over other pipe forming methods. The factors affecting impregnation and degassing are pressure, vibration, temperature and materials. In the present study effects of these factors on impregnation-degassing time were experimentally studied and the approximate speed of impregnation and degassing was theoretically analysed assuming a simple mathematical model.

Results:

(1) It was experimentally clarified that the short impregnation and degassing time characteristic of the centrifugal forming method is due mainly to the enhancement of bubble buoyancy by the centrifugal force and the dither effect of mechanical vibration.

(2) Speed of impregnation and degassing was mathematically analysed by setting up a simple model reflecting the experimentally observed mechanisms.

(3) The above analysis revealed that the centrifugal force increases the degassing speed but it does not influence the minimum attainable size of bubbles.
\end{abstract}

\section{Introduction}

In a previous paper ${ }^{[1]}$ the authors described the mechanism of the centrifugal fiber winding method in the production of FRP pipes. As a continuation the authors discuss in the present paper the speed of impregnation of resin into fiber and the degassing under the centrifugal force.

FRP (fiber reinforced plastics), one of the earliest compound materials, was first invented by Pittsburg Plate Glass Company as a combination of acrylic resin and glass fiber in 1942. U.S. Rubber Company developed the currently mass produced combination of unsaturated polyester resin and glass-fiber. ${ }^{[2|-| .5 \mid}$ In about 1950, research on cylinder forming of FRP pipes making use of centrifugal force started involving many engineers. However, few published papers ${ }^{|6|-112 \mid}$ are available on this subject. Literatures in this field are mainly patent documents ${ }^{[13],[14]}$ and no academic papers are available on the impregnation and degassing under the centrifugal force.

In view of above background the authors discuss in the present study the mechanism of resin impregnation and degassing under centrifugal force both experimentally and theoretically. In the centrifugal forming of FRP pipes the centrifugal force and mechanical vibration due to the rotation of mold are known to enhance resin impregnation and degassing. In a series of simulating experiments in the present study the centrifugal force was replaced by a vacuum of equivalent effect and the mechanical vibration was reproduced by a vibrator and their effects on the speed of resin impregnation and degassing were observed. In a separate theoretical analysis factors affecting the speed of impregnation and degassing were modeled in an ordinary differential equation which were solved numerically.

\section{Importance of Resin Impregnation and Degassing in the Centrifugal Forming Method}

When the centrifugally formed FRPM (fiber reinforced plastics mortor) structure shown in Fig. 1 is compared with the handlayed up products of the same structure shown in Fig. 2, the latter contains many void holes but almost no holes are observed in the former. This shows the superiority of the centrifugal forming method in the speed of resin impregnation and degassing. However, as shown in Figs. 3 and 4 defects due to insufficient impregnation or degassing can occur even in the centrifugal forming method when the processing time is too short. Since good resin impregnation and degassing are the major advantages of centrifugal forming method, analysis of their mechanism is quite important. 


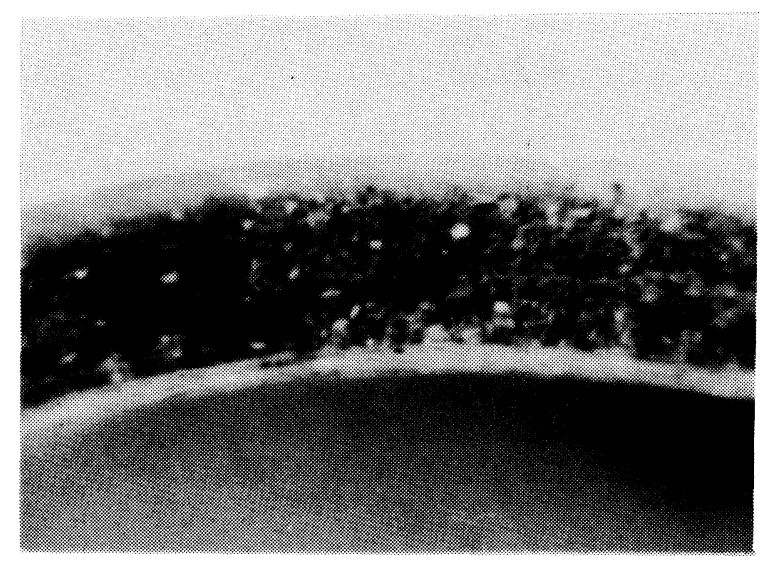

Fig. 1 Centrifugally formed product

Bore: $150 \mathrm{~mm}$; thickness: $12 \mathrm{~mm}$; FRPM sandwich pipe Outer surface FRP layer: Fiber ratio 30 vol\%

Mortar layer: Sand ratio 72 vol\%

Inter surface FRP layer: Fiber ratio 40 vol\%

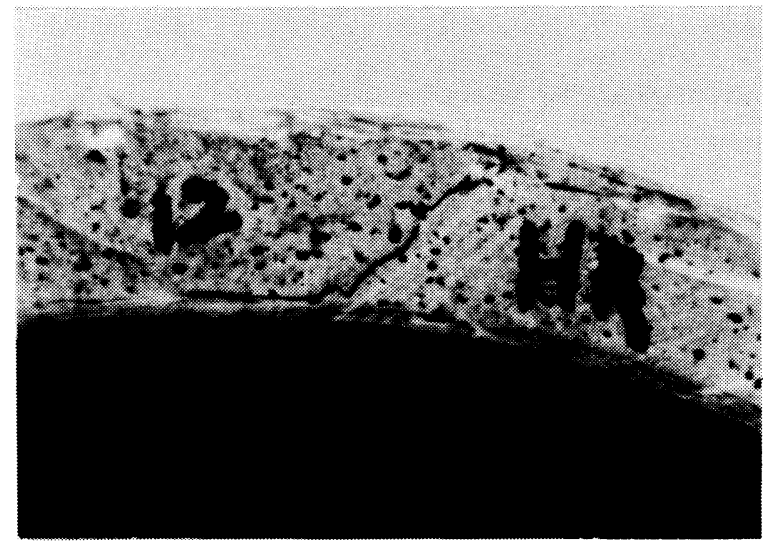

Fig. 2 Hand lay-up formed product

Cracks in the figure were caused by the strength teste.

Dots in the figure represent pinholes.

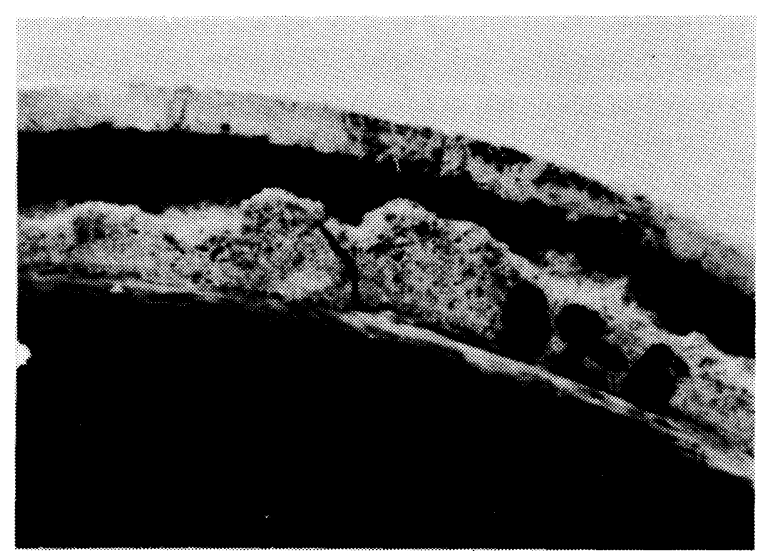

Fig. 3 Insufficient degassing of mortor layer due to improper impregnation

Cracks in the figure were caused by the strength tests.

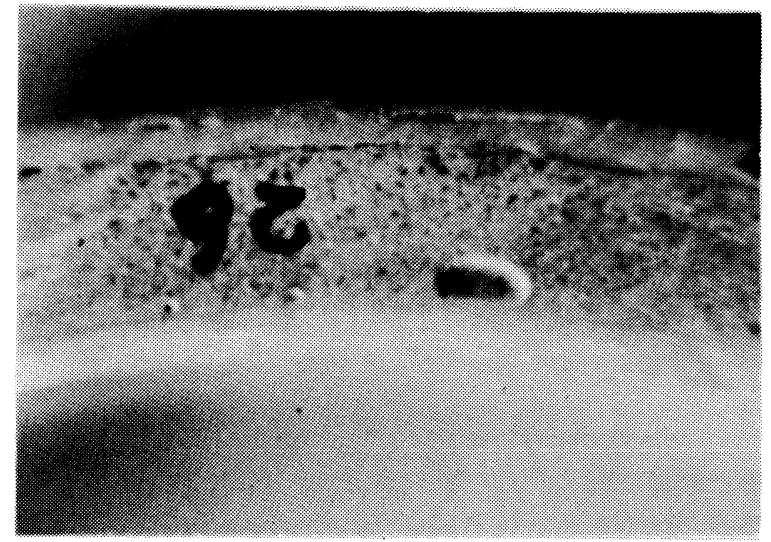

Fig. 4 Void due to improper degassing

Long void on the line on top of the FRP layer is due to insufficient degassing.

\section{Factors Affecting the Resin Impregnation and Degas- sing}

Factors known to influence the impregnation and degassing in the centrifugal forming of FRP pipes are shown in Fig. 5. While

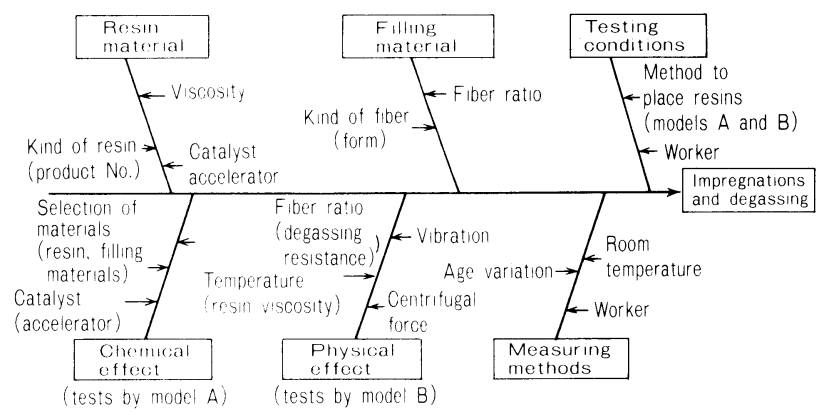

Fig. 5 Factors affecting impregnation and degassing

some factors are physical and others are chemical physical factors play an important role in the centrifugal forming method. Physical factors are centrifugal force, fiber ratio vibration and temperature. Centrifugal force has a strong tendency to separate materials according to their specific gravity. This influences the resin impregnation. Centrifugal force enhances resin impregnation by increasing the buoyancy of air bubbles. Fiber ratio and mechanical vibration influence the resistance to the movement of air bubbles and temperature influence the resin flow by varying its viscosity. Some chemical factors affecting impregnation and degassing are types of resins and fibers, catalysts etc. Since these chemical factors affect the surface activity of materials, they influence the impregnation. Chemical factors can also affect the speed of degassing since they influence the separation of gass bubbles from the fiber reinforcement. It is not difficult to keep other factors than material unchanged in all experiments. Material factors are such as fiber ratio, resin viscosity and surface activity.

\subsection{Simulation test}

Since it was not practical to carry out experiments on a centrifugal pipe forming machine for factors affecting the speed of impregnation and degassing a simulating test using substitute 
Table 1 Testing conditions and items

Total No. of tests: about 450 times

\begin{tabular}{c|c|c|c|c|c}
\hline \hline & $\begin{array}{c}760(\mathrm{mmHg}) \\
\{101.3 \mathrm{kPa}\}\end{array}$ & $\begin{array}{c}560(\mathrm{mmHg}) \\
74.7 \mathrm{kPa}\}\end{array}$ & $\begin{array}{c}360(\mathrm{mmHg}) \\
\{48.0 \mathrm{kPa}\}\end{array}$ & $\begin{array}{c}160(\mathrm{mmHg}) \\
\{21.3 \mathrm{kPa}\}\end{array}$ & $\begin{array}{c}10(\mathrm{mmHg}) \\
\{1.3 \mathrm{kPa}\}\end{array}$ \\
\hline $10\left({ }^{\circ} \mathrm{C}\right)$ & & 0 & $\bigcirc$ & $\bigcirc$ & $\bigcirc$ \\
\hline $20\left({ }^{\circ} \mathrm{C}\right)$ & & $\bigcirc$ & $\bigcirc \square \diamond$ & $\bigcirc$ & $\bigcirc$ \\
\hline $30\left({ }^{\circ} \mathrm{C}\right)$ & & $\bigcirc$ & $\bigcirc \square \diamond$ & $\bigcirc$ & $\bigcirc$ \\
\hline $40\left({ }^{\circ} \mathrm{C}\right)$ & $\square[$ & $\bigcirc \square \square$ & $\bigcirc \square \square \square \diamond$ & $\bigcirc \square \square \square \diamond$ & $\bigcirc$ \\
\hline
\end{tabular}

(Resin) R1: 8225APs; R2: 3308A; R3: 3702-TAKEDA

R4: TD-R-1068; R5: J-8029; R6: 8011; R7: 8027-DAINIHON INK

(Fiber) Glass tape, B11B-ASAHI FIBER; G2: Roving cloth WA570B;

G3: Roving RS240E-601BE-NITTOBO

(Catalyst) BPO 1\% (mark: Existence of catalyst)

Mark $\bigcirc$ : Combination of R1-R7, and G1-G3 in model A all

$\square$ : Model B with R1, G2

: Vibrated model B with R1, G2 (strong)

D : Same as above (weak)

$\diamond$ : Model B under vibration and compression

factors was attempted. Effects of centrifugal force on the speed of impregnation and degassing was simulated in the following manner. Impregnation is expected to be influenced by the difference between resin and fiber in specific gravity. To confirm the effects of specific gravity difference experiments were carried out in two different modes $\mathrm{A}$ and $\mathrm{B}$. In A fiber reinforcement was placed on the fluid resin and in B the resin was poured over the fiber. Degassing is influenced by the buoyancy acting on air bubbles. On way to simulate the effect of buoyancy is to use a fluid having a viscosity close to the resin but much higher in density so that a big static pressure gradient is generated, to simulate the effect of centrifugal force. Such a fluid, however, has the difficulty of floating the fiber. After considering the pros and cons of different schemes it was finally decided to simulate the effects of centrifugal force by the application of vacuum since vacuum enlarges air bubbles to increase their buoyancy to result in a shortened degassing time like the centrifugal force does.

The effect of the vibration due to the revolution of mold was simulated by placing the resin-fiber specimen on top of a mechanical vibrator and pressing a wire brush against the laminated layer from above. Factors affecting the compatibility between fiber and resin (chemical surface active effect) such as material type, catalysts, temperature,etc., were tested in experiment $\mathrm{A}$ to be discussed in detail later. Experimental conditions such as temperature and vacuum are summarized in Table 1. A fixed quantity of resin was used throughout the experiments listed in Table 1, an equivalent of $4 \mathrm{~mm}$ thick laminated layer. Different fibers were used in different quantity but the lamination layer thickness was always made about $1 \mathrm{~mm}$. Hereafter fiber ratio is defined as the one measured in air unless specified otherwise. The number of piles and fiber density were: 1 ply (about $25 \%$ vol) with GL, 3 ply (about $25 \%$ vol) with G2, and 1 ply (about $10 \%$ vol) with G3. The resins were commercially available unsaturated polyester resins (containing about $40 \%$ wt styrene) $4 \sim 14 \mathrm{p}\{0.4-1.4 \mathrm{~Pa} \cdot s\}$ in viscosity. The reason why such a low fiber filling density (fiber ratio) was selected was that a high fiber ratio results in prolonged experiment time and unclear end point of impregnation and degassing. BPO was used as a catalyst to eliminate the undesirable effects of resin bardening.

\subsection{Test equipment and test procedure}

Figure 6 shows the schematic of impregnation and degassing

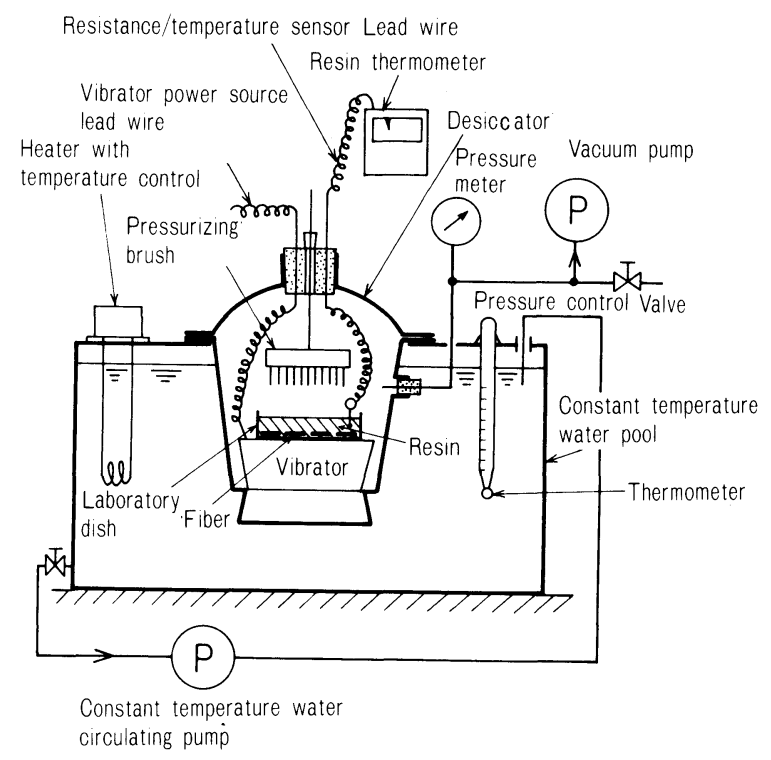

Fig. 6 Impregnation and degassing test equipment

tests. A desiccator is placed in a constant temperature water pool. A vacuum line is connected to the desiccator. Placed inside the desiccator are an electric vibrator (weak: about $1,200 \mathrm{~mm}^{-1}$, in frequency and $0.2 \mathrm{~mm}$ in amplitude, strong: about $1,700 \mathrm{~min}^{-1}$ in frequency and about $0.3 \mathrm{~mm}$ in amplitude), a laboratory dish for specimens attached on top of the vibrator with a doublefaced adhesive tape and a wire brush (wooden bandle brush of about $0.2 \mathrm{~mm}$ in wire dia. and about $20 \mathrm{~mm}$ in wire length) was pressed against the specimen by means of a dead weight plumb [small: about $5 \mathrm{gf} / \mathrm{cm}^{2}\{490 \mathrm{~Pa}\}$, large: about $\mathrm{gf} / \mathrm{cm}^{2}\{1470 \mathrm{~Pa}\}$ ]. Test procedure is as follows.

Test A:

The resin in a laboratory dish was made to reach a predetermined temperature added with a catalyst (BPO, $1 \mathrm{wt} \%$ ) when required, stirred well and the fiber was placed on the resin. Then, 
the inside of the desiccator was decompressed. It was judged the impregnation was completed when the air bubbles became independent as shown in Fig. 7 and the degassing was judged completed when the air bubbles disappeared as shown in Fig. 8 . The elapse of time after the fiber is placed on the resin until

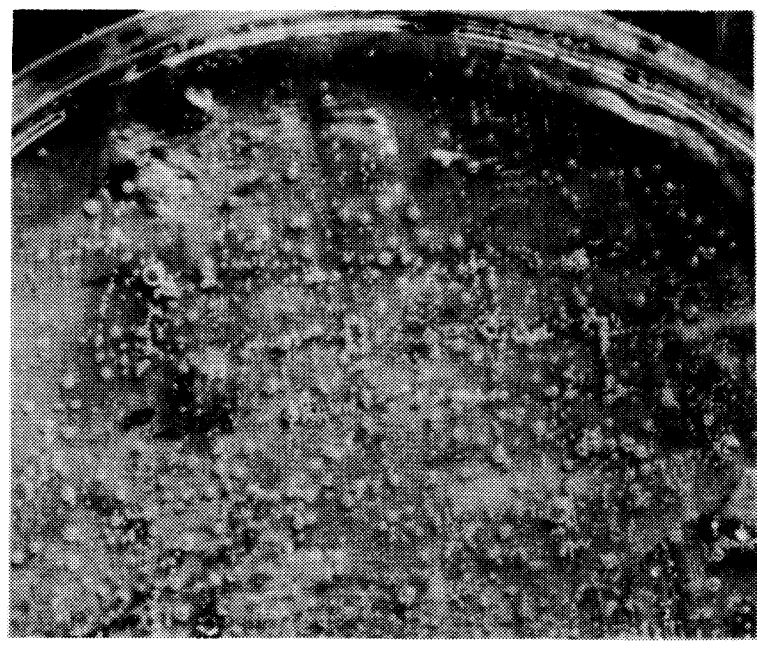

Fig. 7 At completion of impregnation

Resin: R2

Fiber: G3

Model A

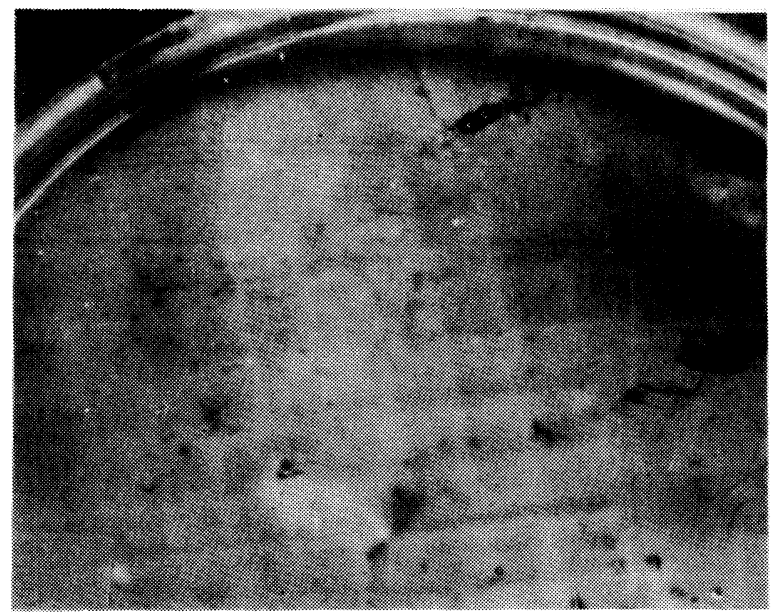

Fig. 8 At completion of impregnation and degassing

Resin: R2

Fiber: G3

Model A

the completion of the impregnation was measured as the impregnation time and the time elapse between the completion of the impregnation to the completion of the degassing was measured as the degassing time. Then the desiccator was returned to the normal pressure, the specimen dish was taken out and its photographs were taken on black paper as the one is Fig. 8 .

\section{Test B:}

Fibers was put in the laboratory dish to cover its whole area. Resin kept of a certain temperature was poured on top of the fiber, pressure inside the desiccator was reduced and the wire brush was pressed against the fiber layer. A certain pressure was given to the pressurizing brush (high pressure and low pressure). Visual inspection was used and photographs were taken and impregnation time and degassing time were measured while the specimen was subjected to mechanical vibration (strong and weak).

\subsection{Test results and discussion}

A. Comparison of Tests A and B

Figure 9 compares Tests $\mathrm{A}$ and $\mathrm{B}$ for impregnation-degassing

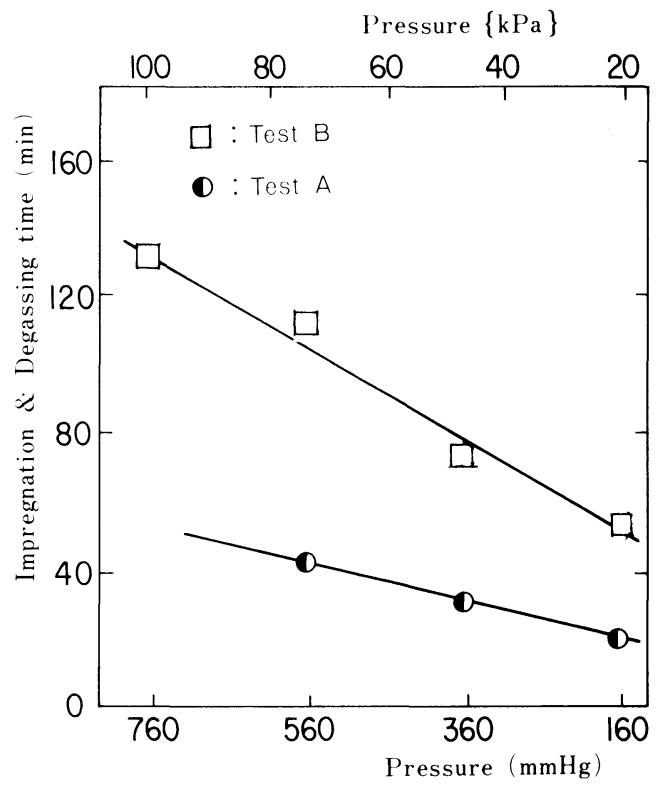

Fig. 9 Comparison of tests A \& B

Resin: R1

Fiber: G2

Temperature: $40^{\circ} \mathrm{C}$

time. Impregnation-degassing time is up to $60 \%$ shorter in Test $\mathrm{A}$ in which the fiber was placed on top of the resin than in Test B in which the resin was poured on top of the fiber layer. In both Tests $A$ and $B$ vacuum enhanced degassing. The difference in time between Test A and B is mainly due to the impregnation time, because the degassing time for Test $B$ was almost the same as in Test A. Main difference between A and B is in the sinking of fiber in resin versus resin sinking in fiber. Specific weight of resin and fiber are respectively 1.1 and 2.6. When the fiber sinks into resin in Test $\mathrm{A}$ the driving force of sinking is the difference $2.6-1.1=1.5$ in specific gravity. Whereas the driving force in Test B is only 1.1 which is about $30 \%$ less than the 1.5 above.

B. Influence of material and catalyst on impregnation-degassing time

Figures 10, 11 and 12 show the influence of fiber, resin and catalyst on the impregnation-degassing time. As shown in Fig. 10 fiber G2 highest (about 25 vol\%) in fiber ratio took the longest impregnation-degassing time. Whereas fiber G3 lowest (about $10 \mathrm{vol} \%)$ in fiber ratio was about $70 \%$ less in impregnationdegassing time. Clearly the fiber ratio influences the impregnation-degassing time. However, since the impregnation and degassing time was shorter in G1 (about 25 vol\%) than in G2 in spite of the similar fiber ratio it is not the only factor influencing the impregnation-degassing time. Fiber surface treatment for increased surface activity seems to have a big influence. In these tests, no pressure or load was exerted on the fiber from outside and therefore the apparent fiber density must have maintained the same level as in dry state.

Figure 11 shows that impregnation-degassing time can differ up to about $30 \%$ for resins of same type and same viscosity $4 \mathrm{P}^{*}$ (about $\{0.4 \mathrm{~Pa} \cdot \mathrm{s}\}$ ). Though not shown in Fig. 11 the impregna- 


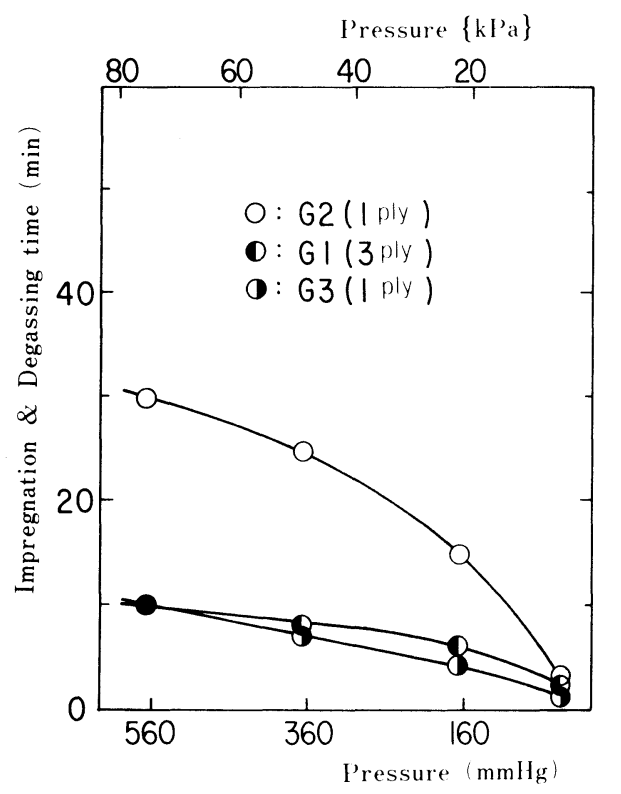

Fig. 10 Influence of fiber

Resin: R1

Temperature: $40^{\circ} \mathrm{C}$

Test: A

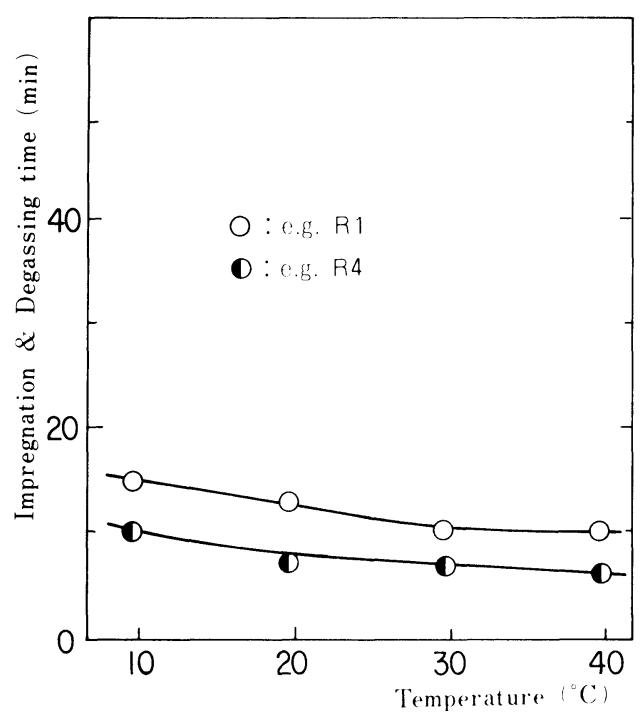

Fig. 11 Influence of resin Fiber: G3

Pressure: $360 \mathrm{mmHg}\{48.0 \mathrm{kPa}\}$

Test: A

tion-degassing time was shorter with resin $\mathrm{R} 1$ (containing an accelerator) about $4 \mathrm{P}$ (about $\{0.4 \mathrm{~Pa} \cdot \mathrm{s}\}$ ) in viscosity than with resin $R 2$ made by the same firm as $R 1$ and lower in viscosity (3P, about $L^{7}$. $\left.3 \mathrm{~Pa} \cdot \mathrm{s}\right\}$ ) than R1. These facts indicate that the surface activity of fiber and resin affect the resin impregnation considerably. Similarly, Fig. 12 indicates that the addition of catalyst has a slight influence on the impregnation-degassing time. This may be an effect of surface active agent contained in the catalyst, or the influence of peroxide catalyst, itself.

C. Influence of vacuum

Figure 13 shows the effect of vacuum on the impregnationdegassing time. The impregnation-degassing time is reduced by up to about $30 \%$ when the pressure is lowered from $560 \mathrm{mmHg}$ $\{74.6 \mathrm{kPa}\}$ to $360 \mathrm{mmHg}\{48 \mathrm{kPa}\}$ and by up to about $60 \%$ when

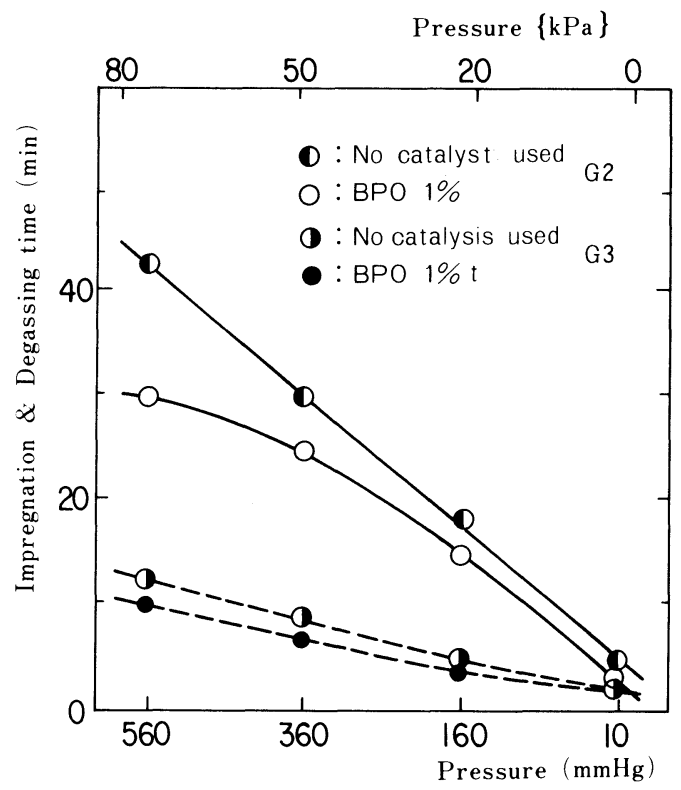

Fig. 12 Influence of catalyst

Resin: R1

Temperature: $40^{\circ} \mathrm{C}$

Test: A

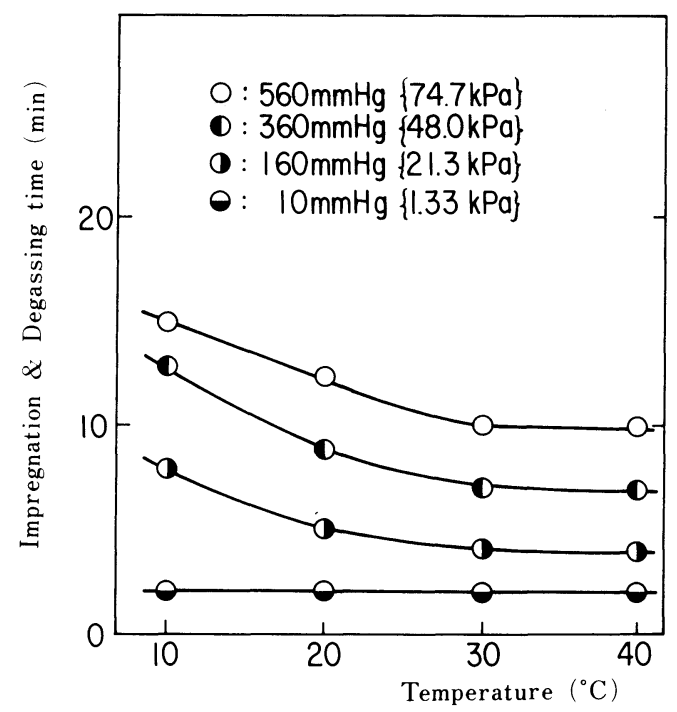

Fig: 13 Influence of vacuum and temperature Resin: R1

Fiber: G3

Test: A

the pressure is further reduced to $160 \mathrm{mmHg}\{21.3 \mathrm{kPa}\}$. Presumably the apparent increase in buoyancy due to the expansion of air bubbles caused by the vacuum accelerates the degassing. The impregnation-degassing time greatly decreases at a pressure between $160 \mathrm{mmHg}\{21.3 \mathrm{kPa}\}$ and $10 \mathrm{mmHg}\{1.3 \mathrm{kPa}\}$ since the styrene monomer starts to boil in this pressure range to greatly enhance the degassing. This is not a mechanical degassing effect. Besides this pressure range does not concern the centrifugal pipe forming process. Approximate boiling pressure of styrene monomer is $50-100 \mathrm{mmHg} 6.7-13.3 \mathrm{kPa}$ at $20-40^{\circ} \mathrm{C}$.

D. Influence of temperature

The relation between temperature and impregnation-degassing time is shown in Fig. 13. Impregnation-degassing time was reduced by about $20 \%$ when the temperature is raised from $10^{\circ} \mathrm{C}-$ 
$20^{\circ} \mathrm{C}$ and by about $40 \%$ at $30-40^{\circ} \mathrm{C}$. This is because the rise of temperature reduces the resin viscosity as shown in Fig. 14. On

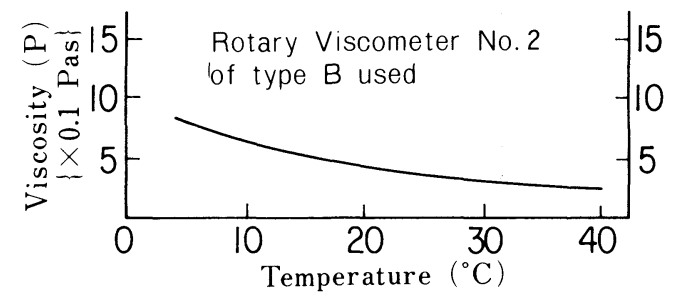

Fig. 14 Temperature versus viscosity for $\mathrm{Rl}$ resin

the other hand when the styrene monomer is boiling impregnation-degassing time becomes independent of temperature.

\section{E. Influence of mechanical vibration}

Figure 15 shows the effects of vibration and wire brush pressing upon impregnation-degassing time. A weak vibration had little effect on impregnation and degassing but when the vibration was strong the impregnation and degassing time was reduced by up to about $60 \%$. This is presumably because the vibration aids the movement of air bubbles through fiber layers. It was also found that the vibration must have a certain minimum strength before it can be effective.

F. Compounded effects of vibration and wire brush pressing

Figure 15 shows that the impregnation-degassing time is not

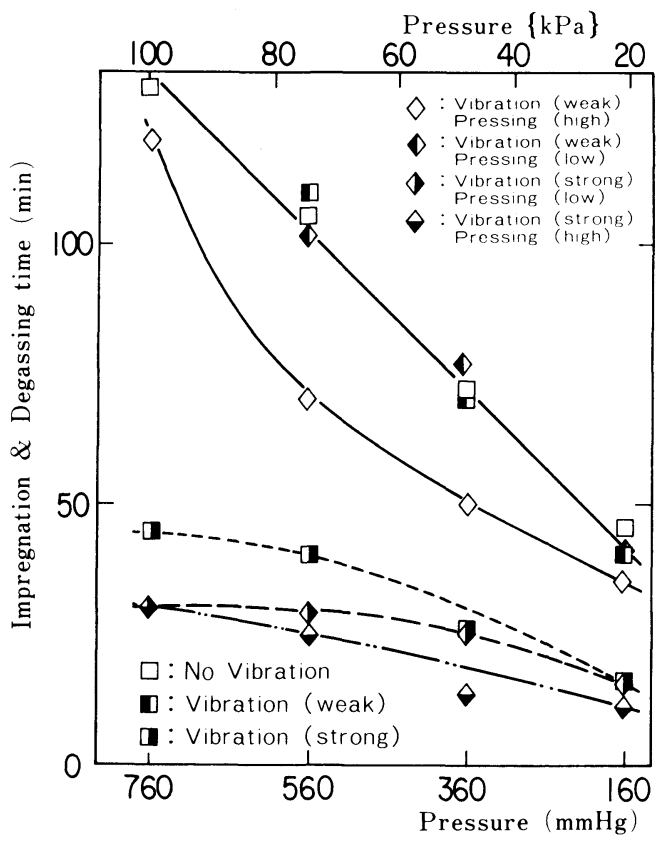

Fig. 15 Influence of vibration and wire brush pressing Resin: R1

Fiber: G2

Temperature: $40^{\circ} \mathrm{C}$

Testing model: $\mathrm{B}$

affected by wire brush pressing when the pressing was weak. When the pressing was strong impregnation-degassing time was reduced by up to about $10 \%$. When the vibration was strong a weak wire brush pressing reduced the time by up to about $5 \%$ and a strong pressing up to about $10 \%$. In other words influence of pressurization was generally small. In the experiments shown in Fig. 15 minimum impregnation-degassing time was obtained in Test $\mathrm{B}$ at temperature $40^{\circ} \mathrm{C}$, vacuum $(160 \mathrm{mmHg}\{21.3 \mathrm{kPa}\}$, vibration (strong) and wire brush pressing (strong). The impregnation-degassing time at the above conditions was about $10 \%$ of that at temperature $40^{\circ} \mathrm{C}$ and normal pressure $(760 \mathrm{~mm} \mathrm{Hg}$ $\{101.3 \mathrm{kPa}\}$ ).

\subsection{Factors affecting the impregnation and degassing in centrifugal forming}

Figure 16 shows the extent to which each factor can affect the

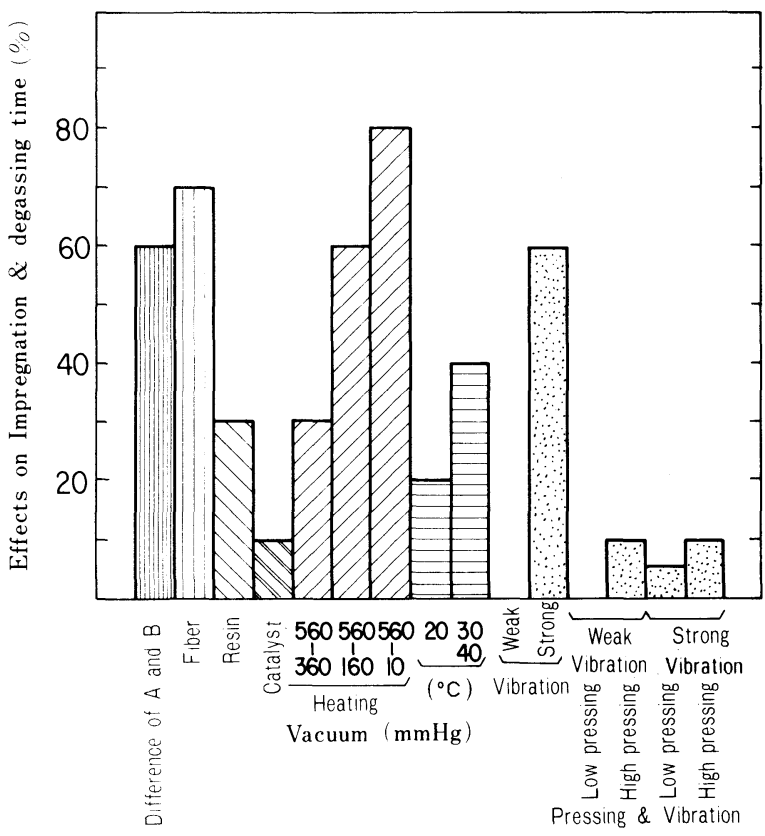

Fig. 16 Effects of different factors on impregnation-degassing time: largest of effects as shown in Figs. 9-15

impregnation-degassing at the most. Written under the horizontal axis are the extent by which each factor was varied. The percentage graduated on the vertical axis is the maximum impregnation-degassing time minus its minimum divided by the maximum source data are as shown in Fig. 9 to Fig. 15. Changing from Test $\mathrm{A}$ to $\mathrm{B}$ reduces the impregnation-degassing time by about $60-70 \%$ and the effect of fiber variety comes to about the same extent. Among chemical factors effect of resin variation was about $30 \%$ and the presence and absence of catalyst $10 \%$. Disregarding the insignificant effect of catalyst, the influence of varying surface activity due to resin type seems to be about $30 \%$.

Factors such as the difference between Tests A and B and selection of materials influence mainly the impregnation through changing the surface activity.

As for the physical factors, the effect of vacuum is as large as $80 \%$, the effect of vibration is $60 \%$ and the effects of heating is $40 \%$. But the effect of pressing with wire brush is as small as $10 \%$. The above found evidences should be applicable to the impregnation and degassing in the centrifugal forming of FRP pipes.

Figure 16 shows the effects of each factor in the unit of percentage. While these values correctly indicate the effect of individual factors they do not necessarily reflect the relative importance of each factor in the centrifugal forming of FRP pipes. At this point one comment on the effect of fiber ratio may be made. In above model experiments fiber ratio or density was invariably low. Whereas in industrial forming of FRP pipes a 
much higher fiber ratio is used. When fiber ratio is high physical resistance of fiber layers in impregnation is estimated to become a predominant factor. Under such circumstances the relative importance of surface activity due to chemical factors is expected to become diminished.

\section{Mathematical Modeling of Impregnation and Degassing Time in the Centrifugal Forming Method}

From above test results, following are considered as major factors affecting the impregnation and degassing in centrifugal pipe forming resistance due to fiber layer, increase of air bubble buoyancy due to the centrifugal force, resin viscosity affected by temperature and mechanical vibration of mold. However, it is difficult to solve the extent of impregnation and degassing as a time function since the following factors are not well defined.

- Effect of mechanical vibration and fiber ratio or density on the resistance to the movement of air bubbles during degassing.

- Quantitative estimation of the effect of surface activity between fiber and resin.

- Developments during the hardening of resins.

In spite of above listed obstacles it is still possible and worthwhile to develop a simplified model which can approximately estimate the conditions required to bring the diameter of residual air babbles less than a certain tolerance. Discussed in what follows are attempts at such a modelling.

\subsection{Assumptions and theories}

It was discussed in section 3.3.A that the driving force of resin impregnation is the buoyancy or the difference in specific gravity. In Test A fibers sink into the fluid resin. The driving force in this case is the specific weight of fiber minus that of the resin $(2.6-1.1=1.5)$. Whereas in Test B trapped air must travel to the fluid surface making the driving force $(1.1-0=1.1)$. The driving force is by $1.5 / 1.1$ in favor of Test $\mathrm{A}$. In industrial centrifugal pipe forming process, however, the centrifugal acceleration is in the very high range of $10-100 \mathrm{~g}$. Under such a high acceleration resin impregnation most probably completes in a very short time. Test $\mathrm{A}$ and Test $\mathrm{B}$ resin impregnation modes as shown in Fig. 17 have their counter parts in industrial centrif-

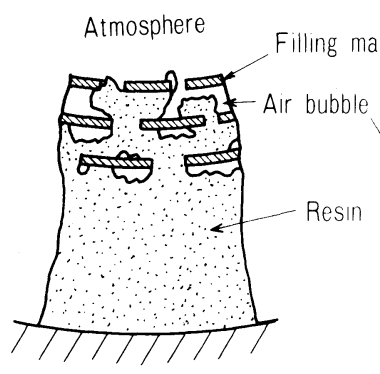

Test A impregnation method

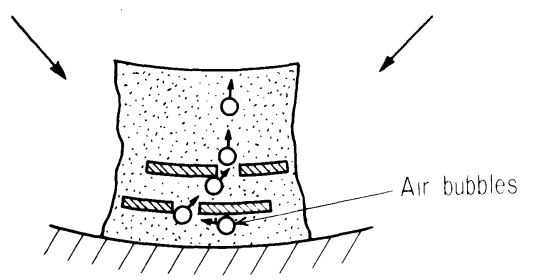

Assumed degassing condition

Fig. 17 Assumed centrifugal impregnation and degassing condition ugal forming process. Under high centrifugal acceleration, however, the difference between Test A and Test B is probably in significant because the resin impregnation time is short in both cases.

Under such circumstances degassing time probably becomes a major concern. In view of this the degassing process is mathematically modeled in the paragraphs to follow (see also Fig. 17). After the completion of the resin impregnation process a number of independent small air bubbles are assumed to remain in the fluid resin. As shown in Fig. 17, the air bubbles should pass through fiber reinforcements. For this reason the maximum air bubble diameter may be considered to be close to the clearance between fibers. When there are air bubbles larger than the clearance between fibers or when much energy is required to dislodge the air bubbles from the fibers mechanical vibration may play a big role in breaking up the bubbles to make them start to move upward.

Another important element in degassing is the buoyancy acting on air bubbles. In the model experiments discussed above buoyancy was increased by subjecting the system to vacuum which greatly reduced the degassing time. A buoyancy increasing test by means of centrifugal acceleration could not be performed. However a large buoyancy enhancement can be expected in the centrifugal forming. In view of above evidences the motion of air bubble while in degassing was modeled in the following manner. The air bubble is subject to viscous resistance of fluid resin while mechanical vibration aids the dislodging of air bubble from fibers. The temperature of resin can be considered to be constant since the thermal capacity of the mold, filling materials etc. are large enough to absorb the heat generated by the resin hardening. Since the air bubbles are filtered by the fiber, layer air bubble diameter is assumed small and almost uniform. Upon such an assumption, the air bubbles can be considered to be spherical in shape because of the low speed and low Reynolds number of $10^{-2}$ for the resins used and the small Weber number of less than 1. From above, the resistance acting on the air bubble can be approximately described by the sum of viscous resistance $R$ or resin and the resistance $C$ exerted by fiber as shown in Fig. 18.

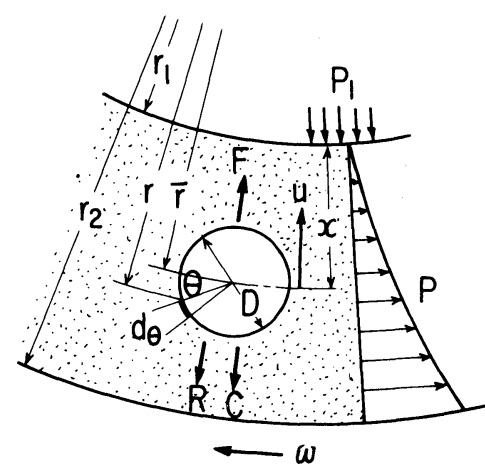

Fig. 18 Relation of forces acting on air bubble

Above assumptions can be summarized as follows.

1) The impregnation process is neglected.

2) Initial diameter of air bubble is almost constant and much smaller than the thickness of the resin layer. Air bubbles are in spherical form.

3) The movement of air bubbles is slow.

4) The fluid resin is everywhere uniform in viscosity.

5) The thickness of resin layer is small in comparison to the 
radius of metal mold.

6) Resistance of fibers to the movement of air bubble is a constant unaffected by bubble radius and location.

7) Surface activity is ignored.

8) There are enough clearances between fibers for the air bubbles to pass through.

The balance of forces acting on an air bubble as shown in Fig. 18 can be described by eq. (1) below.

$$
m \frac{d u}{d t}=F-R-C
$$

where

$m$ : mass of air bubble

$u$ : ascending speed of air bubble (the speed toward the pipe center is assumed to be positive)

$F$ : Buoyancy of air bubble

$R$ : Viscous resistance of resin

$C$ : Resistance exerted by fibers to air bubble.

The pressure $P$ due to centrifugal force can be described by eq. (2) derived by taking the equilibrium of forces acting on a minute hexahedron within the resin layer.

$$
P=P_{1}+\frac{\rho_{R}}{2} \omega^{2}\left(r^{2}-r_{1}^{2}\right)
$$

where

$P_{1}$ : Atomospheric Pressure

$\rho_{R}:$ Resin density

$\boldsymbol{\omega}$ : Angular velocity of metal mold

$r$ : Radial position

$r_{1}$ : Radium to resin surface

Assuming the air bubble to be spherical in shape the buoyancy $F$ acting on the bubble can be expressed by eq. (3) below (also see Fig. 18).

where

$$
F=\int_{A} P \sin \theta d \theta A=\frac{x}{6} D^{3} \omega^{2} \rho_{R} \bar{r}
$$

$A$ : Surface area of air bubble

$\theta$ : Angle from the center of the air bubble

$r$ : Radial position of air bubble

$D$ : Air bubble diameter

Since air bubbles are small sphears moving slowly in viscous fluid the fluid resistance can be described by the Storokes eq. (4).

$$
R=3 \pi \mu D u
$$

where, $\mu$ : viscosity (viscosity at forming temperature).

Velocity $u$ can be expressed as eq. (5) below.

$$
u=-\frac{d \bar{r}}{d t}=-\frac{d x}{d t}
$$

When eqs. (1) to (5) are substituted into eq. (1), eq. (6) approximately describing the centrifugal degassing process can be obtained.

$$
m \frac{d x^{2}}{d t^{2}}+3 \pi D \mu \frac{d t}{d t}+\frac{\pi}{6} D^{3} \rho_{R} \omega^{2} x=\frac{\pi}{6} D^{3} \rho_{R} \omega^{2} r_{1}+C
$$

where, $x$ : depth measured from the free resin surface to the air bubble center

Hereby, the air bubble diameter is assumed constant. The error incurred by this assumption may be estimated by the formula below provided the mechanical vibration does not split the air bubble and the state equation is $P_{L} V=$ constant or $V_{\infty} \quad 1 / P_{L}$.

$$
\therefore \frac{D_{\max }-D_{\min }}{D_{\min }}=\sqrt[3]{\frac{P_{L}+\frac{1}{2} \rho_{R}\left(r_{2}^{2}-r_{1}^{2}\right) \omega^{2}}{P_{L}}}-1 \leqq 1.8 \%
$$

The error is small enough.

In above formula,

$P_{L}$ : Pressure air bubbles in the resin layer

$V$ : Volume of an air bubble

$r_{2}$ : radius of metal mold.

Appropriate values of $\omega, \rho_{R}, r_{1}$ and $r_{2}$ used in above computa- tion are $\omega \leqq 100 \mathrm{rad} / \mathrm{s}, \quad \rho_{k^{\prime}}=1.1 \mathrm{~g} / \mathrm{cm}^{3}, \quad r_{2}-r_{1} \doteqdot 1 \mathrm{~cm}$, $r_{2}+r_{1} \fallingdotseq 10 \mathrm{~cm}, r_{2}-r_{1}=1 \mathrm{~cm}$, and $r_{2}+r_{1}=10 \mathrm{~cm}$

\subsection{Solution of approxiate equations}

If an air bubble $1 \mathrm{~mm}$ in diameter is considered in eq. (6), $m$ and $\mu$ take on the values:

$$
\begin{aligned}
& m=6.66 \times 10^{-7} \mathrm{gf} \cdot \mathrm{s}^{2} / \mathrm{cm} \quad\left\{6.8 \times 10^{-10} \mathrm{~g}\right\} \\
& \mu=1 \sim 80 P\left\{1 \times 10^{-1}-8 \mathrm{~Pa} \cdot \mathrm{s}\right\}
\end{aligned}
$$

Under these parameter values the inertia term on the left hand side of eq. (6) is negligible in comparison to the viscous resistance term. Then eq. (6) becomes a first order differential equation and its general solution is eq. (7) for the initial condition of $x \fallingdotseq$ $T_{L}$ at $t=0$ (see Fig. 18).

$$
x \fallingdotseq\left(\frac{6 C}{\pi I^{3} \omega^{2} \rho_{R}}-r_{1}-T_{L}\right)\left(1-e^{-\frac{D^{2} \rho_{B} \omega^{2}}{18 \mu} t}\right)+T_{L} \quad \cdots(7)
$$

Suppose the bubble reaches the free resin surface $x \fallingdotseq 0$ at $t=$ $t_{0}, t_{0}$ derives from eq. (7) as eq. (8) below.

$$
t_{0}=-\frac{18 \mu}{D^{2} \omega^{2} \rho_{R}} \ln \left\{\frac{D^{2} \omega^{2} \rho_{R} r_{1}-6 \beta q}{\left(T_{L}+r_{1}\right) D^{2} \omega^{2} \rho_{R}-6 \beta q}\right\}
$$

where, $t_{0}$ : degassing time or the time air bubble takes to move from depth $T_{L}$ to resin surface. $\left(t_{0} \max \rightarrow T_{L \max }\right)$

$T_{L}$ : Initial depth of air bubble $\left(T_{L_{\max }} \fallingdotseq r_{2}-r_{1}\right)$

$C$ represents the resistance exerted by fiber layers to the air bubble. For the convenience of modelling it was intended to assign a constant value in the range $0.1-10.0$ to $C$. Next considering the spherical form of air bubble and that the adhesion of air bubble to fiber layer has something to do with surface tension was expressed by the formula

$$
C=\beta \pi D q
$$

where

$\beta$ : coefficient of resistance

$q$ : Surface tension acting on air bubble in resin layer.

Equation (8) is the approximate formula giving the degassing time in the centrifugal pipe forming process.

A simplified form of eq. (8) is obtained as eq. (9) by ignoring the second order terms. Error incurred by this simplification is less than $10 \%$

where

$$
t_{0} \doteqdot \frac{18 \mu T_{L}}{I^{2} \rho_{R} \alpha g f-6 \beta q}
$$

$$
\alpha=\frac{r \omega^{2}}{g f}=\frac{r \cdot \omega^{2}}{g f}=\frac{r i \omega^{2}}{g f}
$$

$\alpha$ : non-dimensional centrifugal acceleration (as multiple of acceleration of gravity)

$g f:$ acceleration of gravity

An air bubble remaining in finished product is a defect when its diameter is larger than a certain allowable value $D_{\min }$.

Furthermore initial depth of the air bubble is assumed to be equal to the deepest possible value $T_{L \max }=r_{2}-r_{1}$ as defined in eq. (8). When the $D_{\max }$ and $T_{L \max }$ are substituted into eq. (9) to take the place of $D$ and $T_{L}$ we obtain eq. (9)' below predicting the practical degassing time $t_{c}$.

$$
t_{c} \doteqdot \frac{18 \mu\left(r_{2}-r_{1}\right)}{D_{\min }^{2} \rho_{R} g f-6 \beta q}
$$

On the other hand, since $t_{0}>0$ in the eq. (8), the quantity inside \{\} on the right hand side must satisfy

$$
\begin{aligned}
& 1 \geqq\left(1-\frac{T D^{2} \omega^{2} \rho_{R}}{\left(T_{L}+r_{1}\right) D^{2} \omega^{2} \rho_{R}-6 \beta q}\right)>0 \\
& \therefore \quad I)>\sqrt{\frac{6 \beta q}{\rho_{R} \alpha g f}} \ldots \ldots \ldots \ldots \ldots \ldots \ldots \ldots \ldots \ldots \ldots \ldots \ldots \ldots
\end{aligned}
$$

The inequality (10) above gives approximately the diameter of smallest removable air bubbles. Hereafter the $D$ given by (10) is called the ultimate degassing range. Shown in Figure 19 are $t_{0}$ versus $\alpha$ curves as given by eq. (9) and (10) for representative $\beta$ and $D$ values. Fig. 20 shows $D$ versus $\beta$ curves under several $\alpha$ 


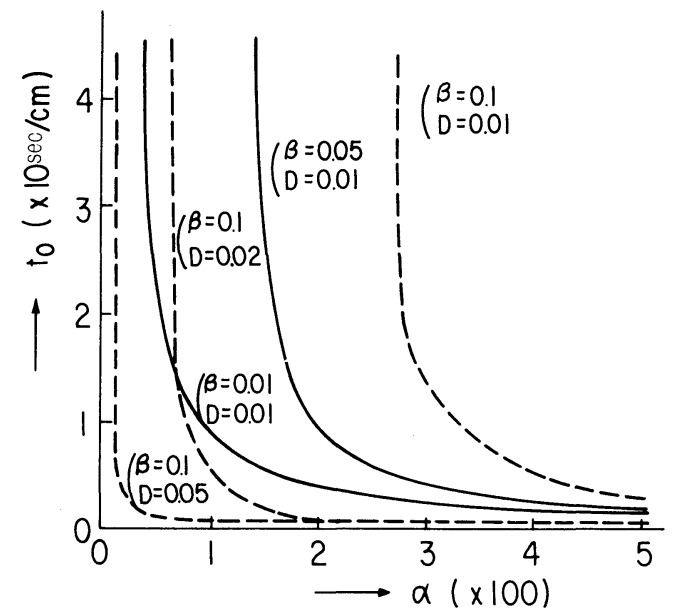

Fig. 19 Relation among centrifugal impregnation and degassing speed, air bubble dia. and resistance

Note: $\rho_{R}=1.1 ; \mu=4 ; q=45$ (c.g.s. unit)

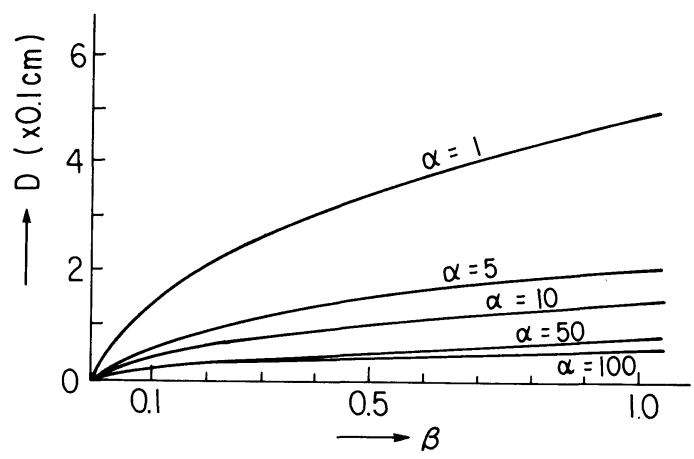

Fig. 20 Relation among possible degassing limit, air bubble dia. and resistance

Note: $t_{0}=\infty, \rho_{R}=1.1, q=45$, (c.g.s. unit system)

values. Figure 19 shows that $\beta$ and $D$ greatly influence the degassing time and that centrifugal force $a$ has to be adjusted to suit the given $D$ and $\beta$ values.

Figure 20 shows that the ultimate degassing limit for intinite degassing time is influenced little by $a$ but largely by $\beta$. In other words, the centrifugal force greatly increases the degassing speed but serves little to improve the ultimate degassing limit, or the minimum attainable air bubble diameter remaining.

\subsection{Effect of centrifugal force on degassing}

From eq. (8), the degassing time $t_{0}$ in the absence of centrifugal force $t_{0}$ ' can be obtained as eq. (11) below.

$$
t_{0}^{\prime}=\frac{18 \mu T_{L}}{D^{2} \rho_{R} g f-6 \beta^{\prime} q}
$$

where $\beta$ ! fiber resistance $\left(\beta<\beta^{\prime}\right)$ under no centrifugal force.

In eq. (11) the relation $\beta$ '> $\beta / \alpha$ holds. Further when " $a$ " is defined as $a=1 / 6 D^{2} \rho_{R} g f$, eqs. (9) and (11) yield eq. (12) below.

$$
\frac{t_{0}^{\prime}}{t_{0}} \fallingdotseq \alpha\left(\frac{a-\frac{\beta}{\alpha} q}{a-\beta^{\prime} q}\right)>\alpha
$$

Equation (12) shows that the centrifugal force expressed in the multiple of the acceleration $g$ of gravity reduces the degassing time by more than the ratio $\alpha$.

\subsection{Theoretical model versus experimental results}

Since it was not possible to conduct experiments on centrifugal degassing the results of the simulation experiments shown in Fig. 13 are compared with the theoretical analysis in sections 4.1 to 4.3. Considering that in the simulation experiments buoyancy was enhanced by the application of vacuum eq. (11) is generalized to take the form of (11)' below taking the state of air into consideration.

$$
t_{0}^{\prime}=\frac{18 \mu T_{L}}{D_{0}^{2} \rho_{R} q f}\left(\frac{P T_{0}}{P_{0} T}\right)^{\frac{2}{3}}
$$

However, eq. (11)' is valid only up to the boiling point of the styrene monomer $\left[P_{0}>100 \mathrm{mmHg}(13.3 \mathrm{kPa})\right]$.

where, $T$ and $T_{0}$ are absolute temperature, $D_{0}$ is air bubble diameter at temperature $T_{0}$ and $P_{0}$ is pressure. As mentioned earlier fiber ratio was invariably low in the above simulation experiments. Taking that into consideration $\beta^{\prime}=0$ at $T_{L}=0.4 \mathrm{~cm}$ was used. Further smallest visible air bubble was assumed to $D_{0}=$ $0.1 \mathrm{~mm}$ in diameter which was used in the calculation. Though the theoretical model neglects the impregnation time it can be assumed that the impregnation and degassing are affected by temperature and vacuum in similar manner. In view of above the effects of temperature and vacuum were evaluated in terms of the ratio of the reduction in degassing time due to the deviation of either temperature or pressure from the standard stage $560 \mathrm{mmHg}$ $\{74.7 \mathrm{kPa}\}$ and $10^{\circ} \mathrm{C}$. Theoretical prediction was made by use of eq. $(11)^{\prime}$. Theory and experiments are compared in Fig. 21. As

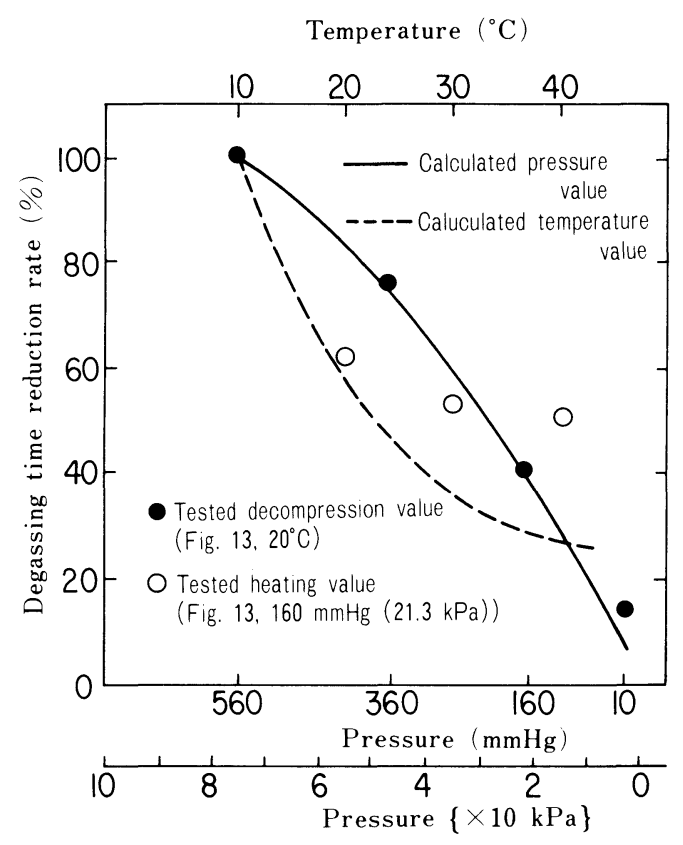

Fig. 21 Comparison between calculation values and test values (Calculated on the basis of $D_{0}=0.01 \mathrm{~cm}, T_{L}=0.4 \mathrm{~cm}, \rho_{R}=1.1$, normal temperature $10^{\circ} \mathrm{C}$, and normal pressure $560 \mathrm{mmHg}\{74.7 \mathrm{kPa}\}$ )

Fig. 21 shows agreement of theory and experiments is good on the effect of vacuum and fair on the effect of temperature. In view of above the theoretical model developed in the present study is an indirect but reasonable simulation of the degassing in the centrifugal pipe forming process.

\section{Conclusion}

By means of indirect simulation experiments factors affecting the degassing time in the centrifugal forming of FRP pipes were found to be centrifugal force, viscous resistance of fluid resin and resistance of fiber layer to the movement of air bubbles. 
A differential equation model was developed to model the above effects.

Other major results are as follow:

(1) Through simulation experiments effects upon degassing of vacuum, temperature, mechanical vibration, fiber content, type of resin and catalysts and the method of resin supply were clarified.

It was found that the centrifugal force and mechanical vibration had the largest effect on enhancing degassing. It was also found that the movement of air bubbles while in degassing was affected by the resistance by the fiber layer and by the temperature dependent viscous resistance of resin.

(2) Theoretical simulation of the degassing process revealed that the centrifugal force measured as the multiple $\alpha$ of the acceleration of gravity reduces the degassing time at least by the ratio $\alpha$. Centrifugal force, however, does not contribute to reduce the smallest attainable bubble diameter. It was found to be affected by $\beta$ rather than $\alpha$.

(3) Future Problems

Further studies are in order to solve the problems below.

- Establishment of experimental method by which the fiber resistance coefficient $\beta$ can be determined more accurately.

- Improvement of the reliability of the approximate theoretical simulation developed in the present study.

\section{References}

[1] Nishino; "Fiber Science Magazine". 32, T57 (1979).

[2] Eiichiro Takiyama; "Polyester Resins". Nikkan Kogyo (1972).

[3] W. D. Qascom et al.; "I \& EC", V7, No. 3 (1968).

[4] J. T. Paul et al.; SPI 20th (1965).

[5] W. Hand; SPI 20th (1965).

[6] Nishino et al.; The Japan Reinforced Plastics Society 26th Symposium 3-1 (1981).

[7] Nishino et al.; The Society of Material Science Japan, 9th FRP Symposium 5 (1980).

[8] Nishino et al.; The Society of Material Science Japan, 9th FRP Symposium 6 (1980).

[9] Nishino et al.; The Textile Machinery Society of Japan, 34th Symposium 401-10 (1981).

[10] Nishino et al.; The Textile Machinery Society of Japan, 34th Symposium 401-11 (1981).

[11] H. Reither et al.; SPI 28th (1973).

[12] T. Kuroda; SPI 19th (1974).

[13] Nishino et al.; The Japan Reinforced Plastics, 26-11 (1981).

[14] Nishino et al.; The Japan Reinforced Plastics, 26-12 (1981).

[15] Busuke Fujimoto; Introduction to Hydraulics, Yookendo. 